LAL 04-15

DAPNIA 04-79

LBNL-54789

May 2004

\title{
First Tests of a Micromegas TPC in a Magnetic Field
}

\author{
Paul Colas ${ }^{\mathrm{a}}$, Ioannis Giomataris ${ }^{\mathrm{a}}$, Vincent Lepeltier ${ }^{\mathrm{b}}$, \\ Michael Ronan ${ }^{\mathrm{c}}$ \\ ${ }^{a}$ DAPNIA, CEA Saclay, 91191 Gif sur Yvette cedex, France \\ ${ }^{\mathrm{b}}$ LAL Orsay, IN2P3-CNRS et Université de Paris-Sud, 91898 Orsay cedex, \\ France \\ ${ }^{\mathrm{c}} L B N L$ Berkeley, CA, USA
}

\begin{abstract}
Since the summer of 2003, a large Micromegas TPC prototype (1000 channels, $50 \mathrm{~cm}$ drift, $50 \mathrm{~cm}$ diameter) has been operated in a $2 \mathrm{~T}$ superconducting magnet at Saclay. A description of this apparatus and first results from cosmic ray tests are presented. Additionnal measurements using simpler detectors with a laser source, an X-ray gun and radio-active sources are discussed. Drift velocity and gain measurements, electron attachment and aging studies for a Micromegas TPC are presented. In particular, using simulations and measurements, it is shown that an Argon- $\mathrm{CF}_{4}$ mixture is optimal for operation at a future Linear Collider.
\end{abstract}

\section{Introduction}

The European TESLA detector and American Large Detector designs include a large Time Projection Chamber (TPC) for the main tracking device. The efficient reconstruction of collimated jets expected at high energy $\mathrm{e}^{+} \mathrm{e}^{-}$colliders requires excellent two-track separation and full coverage of endplane detectors using few-millimeter-width anode pads. To reduce the effect of the severe background conditions, fine granularity readout and low gas sensitivity to neutrons (e.g. a Hydrogen-less mixture) are required, and the chamber must work in a magnetic field of 3 to $4 \mathrm{~T}$. We proposed Micromegas[1] for the amplification stage of the TPC to meet these requirements and to provide a natural suppression of the ion backflow into the drift volume [2]. 
The Berkeley-Orsay-Saclay cosmic-ray setup and the data taking are described in Section 2. In Section 3, we show the Monte-Carlo simulations and experimental studies which favour a new mixture for use in such a TPC. In Section 4 new developments on the expected spatial resolution are addressed.

\section{The Berkeley-Orsay-Saclay cosmic ray setup}

A $50 \mathrm{~cm}$ drift length and $50 \mathrm{~cm}$ diameter TPC, equipped with 1024 electronic channels, has been in operation since July 2003, and has taken magnetic field data in November 2003. The anode is segmented in 8 lines with 96 pads $(2 \times$ $\left.10 \mathrm{~mm}^{2}\right)$, plus 2 central lines of 128 narrower pads $\left(1 \times 10 \mathrm{~mm}^{2}\right)$. The pads are drawn on a Printed Circuit Board (PCB) by standard etching technics. Each pad is read out independently through a metallised via across the PCB. Particular care has been given to the surface quality of the PCB. To sustend the micromesh, $50 \mu \mathrm{m}$ high polyimide pillars, 200 micron diameter, have been formed by etching a photoimageable film. The large copper micromesh has been made at the CERN workshop. The detector is fitted to the bore of a $2 \mathrm{~T}$ superconducting magnet providing a magnetic field, homogeneous at the percent level in the region $\pm 25 \mathrm{~cm}$ each side of the center of the magnet, along the magnet axis.

The signals are amplified, shaped and sampled at the rate of $20 \mathrm{MHz}$ and digitised over 10 bits with the STAR readout electronics. This is the largest micropattern detector ever built. The VME-based data acquisition is triggered at a rate of about $1 \mathrm{~Hz}$ by the coincidence of 2 large scintillators read out by phototubes. The data acquisition conditions were very steady, with mesh

currents less than $0.3 \mathrm{nA}$, no sparking. The data were analysed using Java Analysis Studio and AIDA. Data have been taken with three gas mixtures: $\mathrm{Ar} / \mathrm{CH}_{4}: 90 / 10, \mathrm{Ar} /$ Isobutane : 95/5, $\mathrm{Ar} / \mathrm{CF}_{4}: 97 / 3$. The drift field was 120 $\mathrm{V} / \mathrm{cm}$ in the first case and $200 \mathrm{~V} / \mathrm{cm}$ in the two others. Most tracks were cosmic muons with momentum between $300 \mathrm{MeV}$ and $3 \mathrm{GeV}$, thus minimum ionising and with relatively low multiple scattering.

Prior to building the large setup, we have checked with a small detector with a non-segmented anode that the behaviour of Micromegas was not hampered by a large magnetic field perpendicular to the mesh. We measured the position and the relative width of the $5.9 \mathrm{keV}$ line of an iron 55 source, while varying the magnetic field from 0 to $2 \mathrm{~T}$. They show a remarkable stability as a function of the magnetic field (Figure 1). 


\section{Iron 55 peak position}
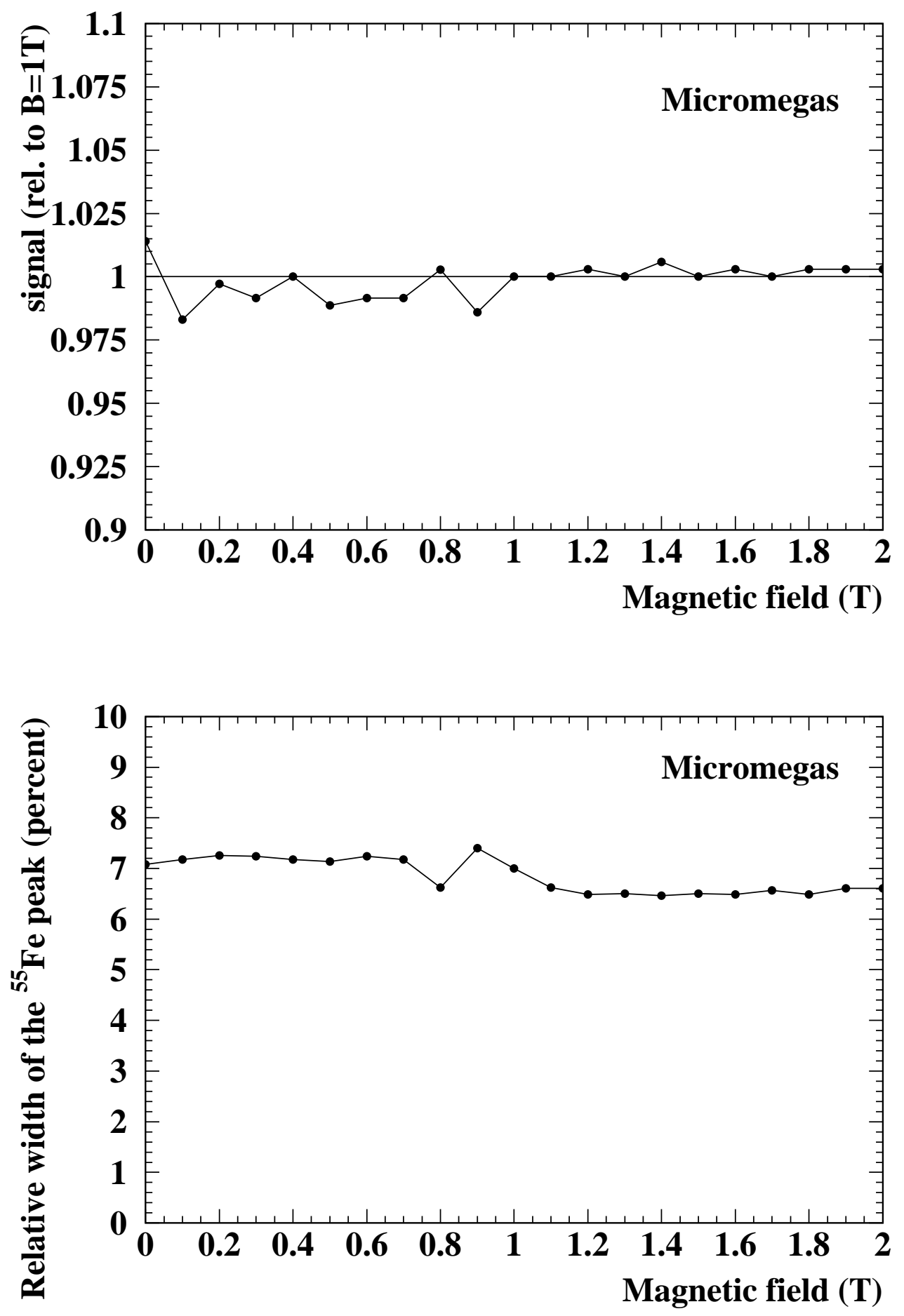

Fig. 1. The peak position relative to $B=1 T$ (top) and the width (bottom) of the ${ }^{55} \mathrm{Fe}$ $5.9 \mathrm{KeV}$ X-ray line measured with a Micromegas, as a function of the magnetic field. 


\section{A magic gas mixture: $\mathrm{Ar}+\mathrm{CF}_{4}$}

Beside demonstrating the feasability and operability of a large scale Micromegas TPC, the goal of the cosmic ray data taking was to confirm Magboltz [3] Monte Carlo calculations of the expected performance of different gas mixtures. Over 50 workable mixtures of a majoritary noble gas (the "carrier") with an admixture (the "quencher") of one or two molecular gases to quench the UV photons produced during the avalanche, have been considered. Simultaneously requiring to have enough primary electrons, have an affordable cost, and present a velocity maximum at low enough field - a field of $200 \mathrm{~V} / \mathrm{cm}$ already requires a $50 \mathrm{kV}$ cathode voltage for a drift length of $2.5 \mathrm{~m}$ - point to Argon as a carrier gas. Most of the quenchers used until now are hydrocarbons. However, Hydrogen nuclei, protons, are bounced by $\mathrm{O}(1 \mathrm{MeV})$ neutrons that are expected to be produced in large numbers by the accelerator and heavily ionise while drifting around magnetic field lines. It is thus preferable to avoid hydrogenated gases. $\mathrm{CO}_{2}$ gives too small drift velocities in mixtures with Ar. In contrast, a few percent of $\mathrm{CF}_{4}$ gives velocities up to $8-9 \mathrm{~cm} / \mu$ s at a low enough field as shown by the curves of Figure 2 .

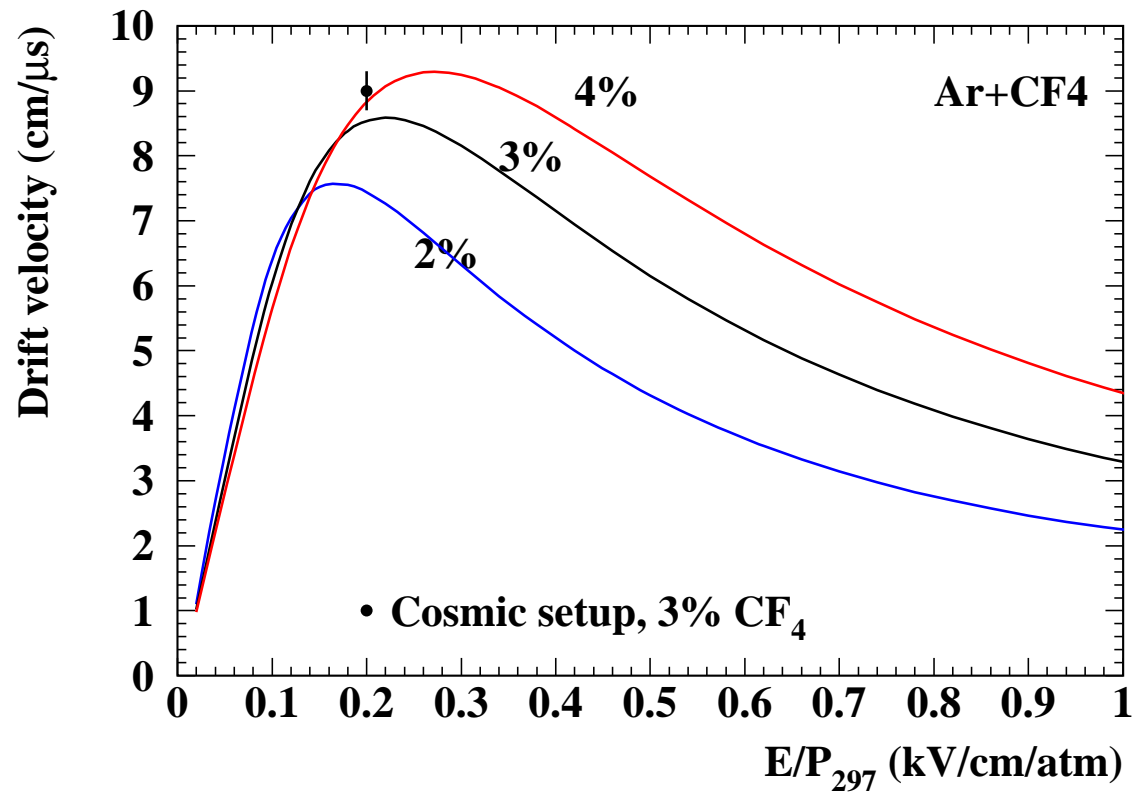

Fig. 2. The drift velocity of electrons as a function of the drift electric field for Ar mixtures with 2, 3 and $4 \%$ of $\mathrm{CF}_{4}$, as predicted by Magboltz. The data point is the measurement with the cosmic ray setup, with $3 \%$ of $\mathrm{CF}_{4}$.

Drift velocity. With the mixture of $\mathrm{Ar}$ with $3 \% \mathrm{CF}_{4}$, the time between the trigger and the arrival of the ionisation buckets on each time cluster was determined. The time distribution presents an edge at 5350 ns with a $3 \%$ accuracy, for a $47.9 \mathrm{~cm}$ drift. This leads to a drift velocity determination of 
$9.0 \pm 0.3 \mathrm{~cm} / \mu \mathrm{s}$ in agreement with the Magboltz prediction of $8.6 \mathrm{~cm} / \mu \mathrm{s}$. As a cross-check, a drift velocity of $4.24 \pm 0.10 \mathrm{~cm} / \mu$ s was measured for an $\mathrm{Ar}+$ $5 \%$ isobutane mixture, where the expectation is $4.16 \mathrm{~cm} / \mu \mathrm{s}$.

Gain. The gain has been measured as a function of the mesh voltage for various concentrations of $\mathrm{CF}_{4}$ in $\mathrm{Ar}$, with a $100 \mu \mathrm{m}$ gap. The result is shown in figure 3. Gains up to $10^{5}$ are reached before sparking. The gain shows an exponential behaviour until a value of 1000, due to secondary effects, and grows faster than an exponential for higher voltages. With existing or foreseeable low-noise electronics, gains as low as 300 or 500 would suffice to operate the detector, and would even be seeked to avoid the formation of space charge in the drift volume. In the cosmic setup, data were taken at a gain of about 800 .

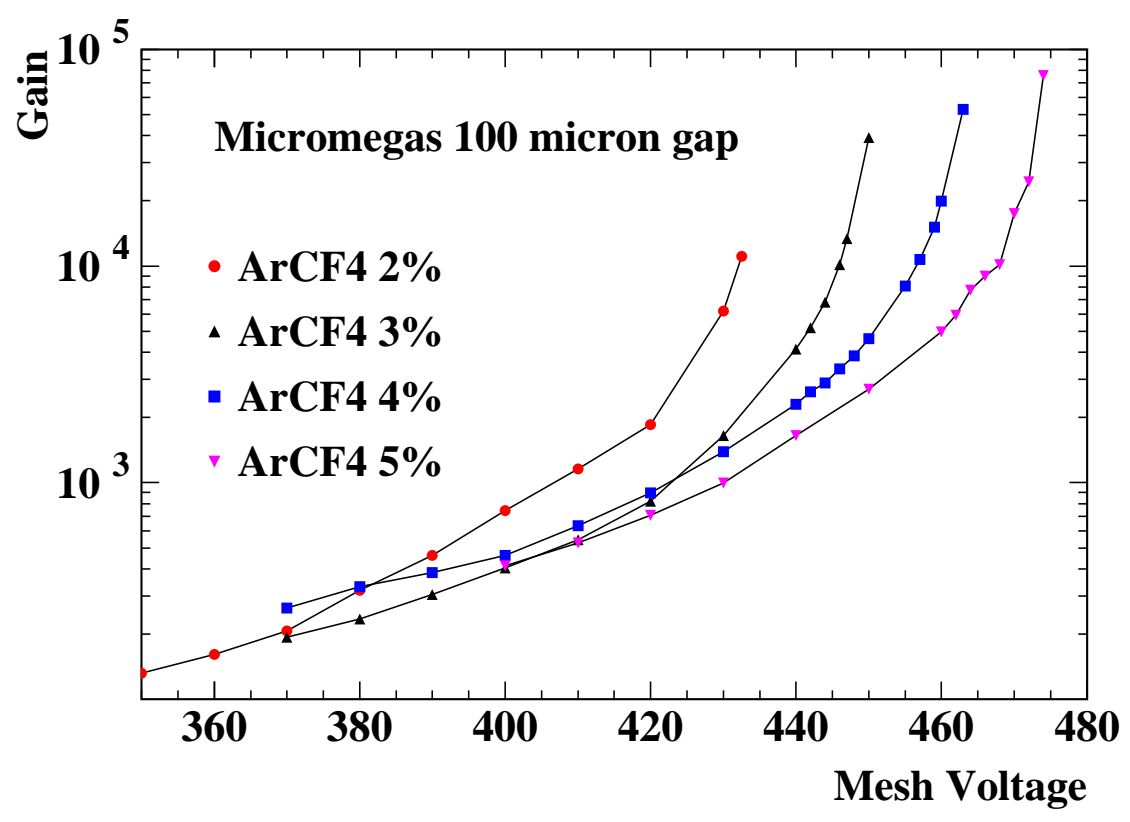

Fig. 3. Gain as a function of the mesh voltage, measured with $a^{55} \mathrm{Fe}$ source and a calibrated amplification chain, for various percentages of $\mathrm{CF}_{4}$ in Argon.

Attachment. There exists a resonance in the attachment cross-section for an electron kinetic energy slightly below the ionisation threshold [4]. This might hamper the operation of a device using this gas, as the negative ions formed are excessively slow. However, the Monte Carlo simulation predicts no attachment (or a negligible attachment) in the drift region, for fields less than 400 $\mathrm{V} / \mathrm{cm}$, and also predicts that the attachment is overwhelmed by the Townsend coefficient in the amplification region. Given the smallness (a few microns) of the transition between the two regions, the operation of Micromegas is not affected by attachment. In the cosmic ray setup, an exponential fit to the truncated mean signal versus drift distance showed no attenuation, allowing a lower limit at $2.4 \mathrm{~m}$ (90\% C.L.) to be set on the attenuation length. 
The dependence of the attachment coefficient as a function of the electric field has been measured from the amplitude of a laser photoelectric signal, in a setup with a $1.29 \mathrm{~cm}$ drift. The data are in excellent agreement with Magboltz predictions (Figure 4).

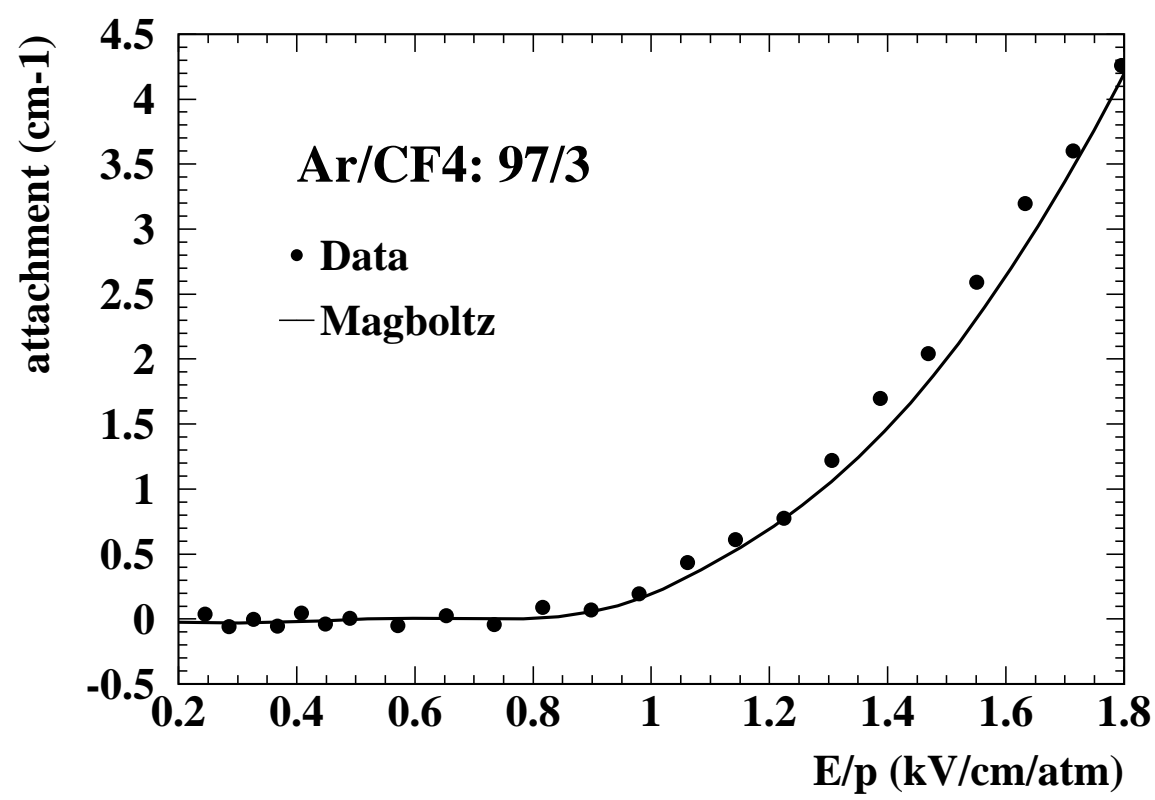

Fig. 4. Attachment coefficient of an Argon (97\%) $\mathrm{CF}_{4}$ mixture as a function of the electric field. The points are data described in the text and the line is the Magboltz Monte Carlo prediction.

Aging. Mixtures containing $\mathrm{CF}_{4}$ have often been convicted of damaging the detectors flushed with them, especially in the presence of Hydrogen atoms and in highly ionising conditions [5]. In the past 5 years however many tests have been carried out with Micromegas detectors with $\mathrm{CF}_{4}$ mixtures, and never signs of degradation have been observed. A dedicated aging test has been carried out at Saclay with an Ar plus $5 \% \mathrm{CF}_{4}$ mixture, by monitoring the mesh current during 3 days, gathering $4 \mathrm{mC} / \mathrm{mm}^{2}$ (over 1,000 years of linear collider with expected background conditions!). The gain was 8000 and no degradation was observed at the percent level. Though the aging issue would require additionnal long-term systematical studies, there is no reason to fear anomalous aging, in well-controlled operating conditions and with a suitable choice of the building materials.

Transverse diffusion. A circle has been fitted to the projection of each track onto the plane transverse to the electric and magnetic fields. Six out of the ten pad rows (including the two with narrow pads) are used in the fit, while the r.m.s. width of the 4 leftover hits is estimated from a maximum likelihood fit to the amplitude distributions as a function of the coordinate along the pad row. Most information comes from the hits consisting of more than 2 pads 
( $50 \%$ of the pads are such) and from tracks near the edge of a single-pad hit. The square of the average of the r.m.s. hit widths $\left(\sigma_{x}^{2}\right)$ for the 1 tesla data sample are plotted versus the drift distance in figure 5 . The linear increase of $\sigma_{x}^{2}$ as a function of the drift distance is characteristic of the diffusion. The slope gives the transverse diffusion constant for Ar plus $3 \% \mathrm{CF}_{4}$ at $\mathrm{B}=1 \mathrm{~T}$ : $\mathrm{D}_{\mathrm{t}}=64 \pm 16 \mu \mathrm{m} / \sqrt{\mathrm{cm}}$ where the error is dominated by systematics, at this preliminary stage of the analysis. This is in agreement with the expectation of $86 \mu \mathrm{m}$. Note that the $\omega \tau$ factor for this fast gas for $\mathrm{B}=1 \mathrm{~T}$ is as large as 4 .

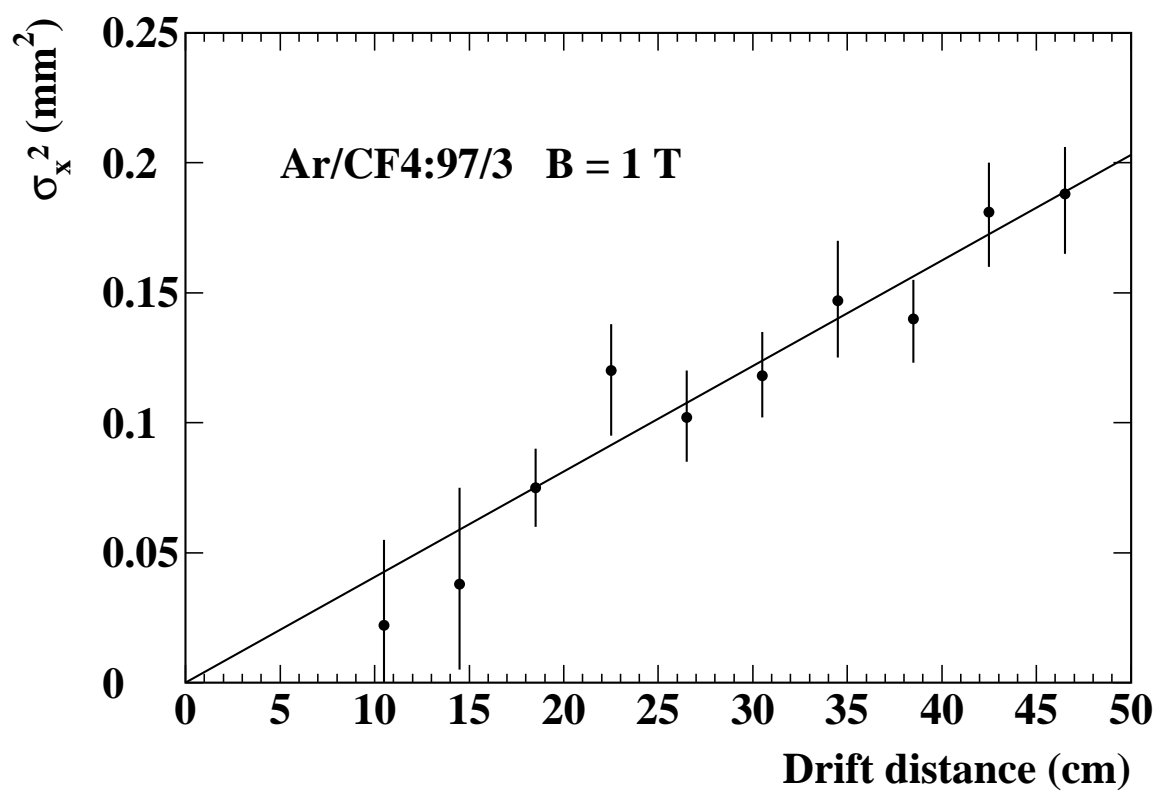

Fig. 5. Track r.m.s. width measured at $1 T$ as a function of the drift distance.

\section{Spatial resolution}

The very low transverse diffusion expected for a LC-TPC operating in magnetic fields of 3-4 $\mathrm{T}$ yields a potential for a very high spatial resolution. However, there is a drawback: at a typical drift distance of $1 \mathrm{~m}$, the r.m.s. width of a track is expected to be $\sim 350 \mu \mathrm{m}$, much smaller than the $2 \mathrm{~mm}$-wide pads that would provide the required 2-track separation. The charge would be collected by a single pad, yielding resolutions of order $600 \mu \mathrm{m}$, much worse than one could expect if one could make a barycenter between 2 or 3 neighbouring pads. Narrower pads would imply an unacceptable increase in the the number of electronic channels. Two solutions have been proposed to this problem.

One possibility is to spread the charge after amplification, by means for instance of a resistive foil [6]. This has been proven to function with GEMs and 
Micromegas and to yield point resolutions of $80 \mu \mathrm{m}$, beyond the requirements for the LC TPC.

Another possibility is the digital TPC [7]: $300 \times 300 \mu \mathrm{m}^{2}$ pads equipped with a digital readout would provide the ultimate resolution and a better $\mathrm{dE} / \mathrm{dx}$ measurement. Practical demonstrations of such devices with a gas amplifier combined with silicon pixels have been presented for the first time at this conference $[8,9]$. This would require $10^{8}$ channels, but all integrated and with a binary (i.e. 1 bit) output.

\section{Conclusion}

A large Micromegas TPC has been operated successfully for the first time in a magnetic field. The first results show that an $\mathrm{Ar}^{\mathrm{CF}_{4}}$ mixture is particularly suited for operation at the linear collider. Obtaining the optimal spatial resolution is still a challenge, but two satisfactory solutions have been recently

demonstrated in principle. At the present stage of the $R \& D$, the adequacy of a Micromegas TPC for the tracking at the linear collider appears thus very promising.

\section{Acknowledgements}

We wish to thank F. Bieser, R. Cizeron, C. Coquelet, E. Delagnes, A. Giganon, G. Guilhem, R. de Oliveira, V. Puill, Ph. Rebourgeard and J.-P. Robert for their help in building and commissionning the detector, and D. Karlen for providing us with the Victoria display and analysis software.

\section{References}

[1] Y. Giomataris, Ph. Rebourgeard, J.P. Robert, G. Charpak, Nucl. Instr. and Meth. A 376 (1996) 29.

[2] P. Colas, I. Giomataris, V. Lepeltier, "Ion back-flow in the Micromegas TPC for the future linear collider", These proceedings, DAPNIA 04-78, LAL 04-14.

[3] S. Biagi, Magboltz program, available from http://www.consult.cern.ch.

[4] S.R. Hunter and L.G. Christophorou, J. Chem. Phys. 80 (1984) 6150.

[5] See for instance: H. Albrecht et al., Nucl. Instr. and Meth. A 515 (2003) 155-165. M. Danilov et al., Nucl. Instr. and Meth. A 515 (2003) 202-219. 
[6] M. S. Dixit, J. Dubeau, J.-P. Martin, and K. Sachs, Nucl. Instr. and Meth. A 518 (2004) 721.

[7] M. Hauschild, "2D and 3D Cluster Counting with GEMs and Small Pads: the Digital TPC?", in Proc. of the International Workshop on Linear Colliders, LCWS02 (August 2002, Jeju, Korea), Ed. J.S. Kang and S.K. Oh, Korean Physical Society, p. 464.

[8] R. Bellazzini, "Reading a GEM with a VLSI pixel ASIC used as a direct charge collecting anode", these proceedings.

[9] P. Colas et al., "The readout of a GEM- or Micromegas- equipped TPC by means of the Medipix2 CMOS sensor as direct anode", these proceedings, CERN-PH-EP/2004-009, DAPNIA 04-77, NIKHEF 2004-3 